\title{
Investigation of Hair Microstructure Changes in Patients with Malignant Tumours by Optical Techniques
}

\author{
Valeriya S. Maryakhina ${ }^{1 *}$, Yulia S. Korneva ${ }^{1,2}$, Maksim V. Ovechkin ${ }^{3}$, Maksim S. Denisov ${ }^{4}$, \\ and Vera I. Spirina ${ }^{5}$ \\ ${ }^{1}$ Smolensk Regional Institute of Pathology, 27 Gagarina str., Smolensk 214020, Russia \\ ${ }^{2}$ Smolensk State Medical University, 28 Krupskoy str., Smolensk 214019, Russia \\ ${ }^{3}$ Orenburg State University, 13 Pobedy str., Orenburg 460018, Russia \\ ${ }^{4}$ A. Tsyb Medical Radiological Research Centre, 4 Koroleva str., Obninsk 249031, Russia \\ ${ }^{5}$ Orenburg Regional Clinical Psychoneurological Hospital for War Veterans, 63 the first May str., Orenburg 460005, \\ Russia \\ *e-mail: valemar@mail.ru
}

\begin{abstract}
The study reports on the differences in hair microstructure of healthy people and patients with cancer. The research has experimentally proved that hair of healthy people has a dense structure with a prominent external layer. In contrast, the hair of cancer patients has a destroyed external layer that is partially absent due to the presence of ulcers. Hair structure defects relate to biochemical changes: cancer presence leads to the increased ratio between oxygen and nitrogen, primarily on account of the decreased nitrogen concentration and, consequently, the decreased peptide concentration. Peptides composition in hair structure was also investigated by fluorimetry. The spectra of fluorescence excitation have several maxima such as at 295, $350 \mathrm{~nm}$. For cancer patients, the spectrum has a huge maximum at $295 \mathrm{~nm}$, while the spectrum for healthy volunteers has a small maximum at the wavelength and is shifting toward shorter wavelengths. We assume that the peak at $350 \mathrm{~nm}$ is primarily defined by the elastin contained in hair. The maximum at $295 \mathrm{~nm}$ is connected with the presence of tryptophan-containing peptides presence, such as keratin extracted from the Henley layer with keratinized cells. The obtained results can be used to improve optical techniques of biomedical diagnostics of cancer diseases. (C) 2019 Journal of Biomedical Photonics \& Engineering.
\end{abstract}

Keywords: cancer; hair; optical biomedical diagnostics; scanning electronic microscopy.

Paper \#3339 received 5 Nov 2019; revised manuscript received 28 Dec 2019; accepted for publication 11 Dec 2019; published online 31 Dec 2019. doi: 10.18287/JBPE19.05.040301.

\section{Introduction}

One of the most important properties of modern techniques in clinical diagnostics is noninvasiveness. It is achieved by either improving the devices or by registration of biochemical properties of cells and tissues, which requires detailed investigation.

It is well-known [1] during tumour growth, the organ undergoes not only local changes. Cancer cells affect host metabolism through the circulating factors they secrete or induce the host to secrete [2]. This leads to disturbance of skin and its appendages [3]. Thus, hair and nails could serve as a health indicator for some diseases that provoke changes in microelement distribution. Most of the published articles contain information about $\mathrm{Ca}$ [4], Mo [5], Fe [6] concentration and their selective accumulation in hair due to the presence of pathological processes. Therefore, the disease may be detected by the analysis of the microelement status of hair [7]. However, these technologies are not widely used because hair microelement status depends on many factors such as nutrition, diseases, shampoos, etc.

In this work, we demonstrate the differences in hair microstructure and their peptide contribution in healthy 
volunteers and cancer patients for the purposes of improving optical biomedical diagnostics.

\section{Materials and methods}

\subsection{Research objects}

The object of this is hair of two groups of volunteers. The first group included 23 healthy women. The second group was composed of women with cancer disease of different localization admitted in the A. Tsyb Medical Radiological Research Centre and the Orenburg Regional Clinical Psychoneurological Hospital for War Veterans. Oncology was confirmed by blood and histological analysis carried out in hospital. Tumour localizations are listed in Table 1. The age of all women was $50 \pm 20$ years for cancer patients and $47 \pm 18$ years for healthy volunteers. All the patients signed the informed consent. The investigation was carried out in conformance with the Agreement of Ethical Committee of the Smolensk State Medical University.

Table 1 Distribution of the cases in the second group for investigation.

\begin{tabular}{cc} 
Diagnosis & Amount \\
\hline Breast cancer & 17 \\
\hline Colon cancer & 3 \\
\hline Melanoma & 2 \\
\hline Lung cancer & 2 \\
\hline Stomach cancer & 1 \\
\hline Pancreas cancer & 1
\end{tabular}

\subsection{Optical microscopy}

After the hair aliquot has been cleared twice in medical alcohol, it was placed on the slide. The hair structure was investigated by means of an Altami Inver 3 optical inverted microscope with a video camera working in the phase contrast mode (magnification $400 \times$ ).

\subsection{Scanning electronic microscopy (SEM)}

The hair microstructure was investigated by means of a JEOL-6000 scanning electronic microscope. Like for the optical microscopy, the cleared hair was placed on the slide. The measurements of microelements (nitrogen and oxygen) distribution and their quantitative analysis were carried out under high vacuum with voltage $20 \mathrm{kV}$.

\subsection{Dynamic light scattering}

The cleared hair aliquot was added to the free-prepared carbonate buffer $(\mathrm{pH}=10)$ for $50 \mathrm{~min}$. To measure the particle size, the obtained suspension was placed to Photocor Compact. The laser wavelength was $647 \mathrm{~nm}$ with the power of $25 \mathrm{~mW}$. All the solutions used were free-prepared from dry compounds produced by SigmaAldrich.

\subsection{Spectrofluorimetry}

The spectra of fluorescence excitation of solutions obtained as for dynamic light scattering were measured by the SOLAR CM-2203 spectrofluorimeter. The wavelength of registration was $410 \mathrm{~nm}$. The spectra were measured in the UV region.

\section{Results and Discussion}

Human hair is known [8] to have a layered structure. Therefore, any changes in metabolic processes lead to changes in the hair biochemical composition and to impact on the layer integrity. As a rule, its destruction starts from the external layer. The photos of the hair samples from healthy volunteers and cancer patients are presented in Fig. 1: the hair of healthy volunteers has a dense structure and a prominent external layer is prominent. In contrast, the hair of cancer patients is thinner with unclear structure. Fig. $1 \mathrm{~b}$ and $1 \mathrm{c}$ depict possible defects of hair structure of cancer patients. Superficial defects in the external layer or its total focal absence are visible in the majority of samples from patients with cancer. The disturbed of geometry and absence of clear structure were observed in several specimens from cancer patients: the hair section has an oval form instead of the round form without scales. We noticed that hair colouring leads to insignificant changes of its structure. The cosmetic effect lasts for a short time (1-2 weeks) whereas the next hair generation gets the structure according to the physiological state of the body.

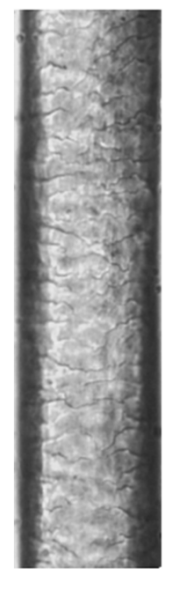

a

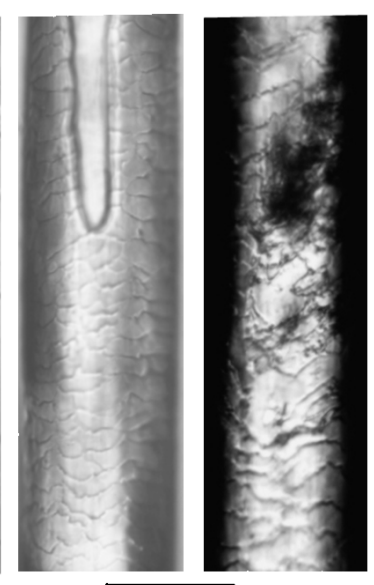

$20 \mu \mathbf{m}$

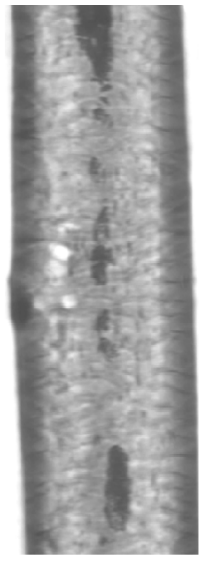

d
Fig. 1 The images of hair in healthy volunteers (a) and cancer patients (b, c, d) carried out by optical microscopy.

Changes in the hair structure are connected to biochemical changes. During the study, the concentrations of nitrogen and oxygen were registered. The results of the measurements are shown in Fig. 2. 
These elements are basic in biological structures and present in all organic compounds. So, nitrogen is a component of amino acids, nucleotides and other vital substances. Concentration of oxygen, in turn, is primarily defined by reactive oxygen species dissolved in a cell and oxygen-containing organic substances.

As can be seen in Fig. 2, concentration of nitrogen decreases due to cancer. This dependence is observed in all samples and is well reproduced. Moreover, concentration of oxygen in the hair of healthy volunteers is lower than in the hair of cancer patients. Cancer cells are known [9] to actively consume peptides and molecular oxygen. As the result of cancer cells metabolism, reactive oxygen species are generated, which leads to cell destruction. Quantitative analysis of nitrogen and oxygen concentrations in the investigated groups depicted in Table 2 suggest that the overlap of weight percentage of oxygen and nitrogen is $100 \%$, while the carbon concentration change is insignificant. The ratio $\mathrm{W}\left(\mathrm{O}_{2}\right) / \mathrm{W}\left(\mathrm{N}_{2}\right)$ is significantly different for these two groups and have statistically reliable means. The presence of malignant tumour leads to the increase of this ratio, on account of the decrease in nitrogen and the consequent decrease in peptide concentration. This dependence was demonstrated in hair samples in mice with spontaneous breast tumour [10].

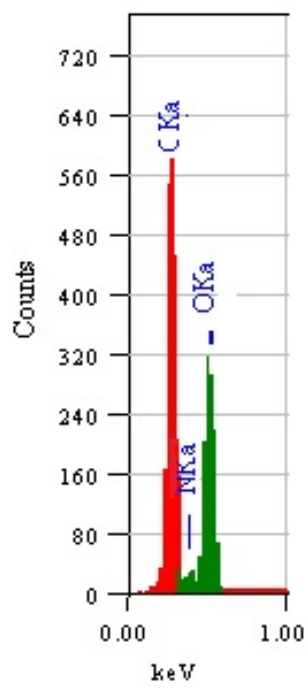

a)

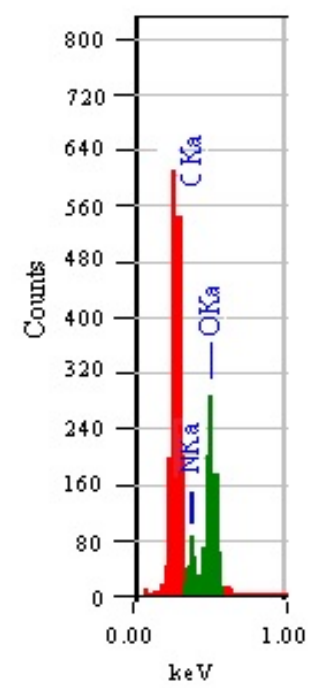

b)
Fig. 2 Typical data of quantitative analysis of carbon, nitrogen and oxygen by SEM in external hair layer of cancer patients (a) and healthy (b) volunteers.

Table 2 The results of quantitative analysis of nitrogen and oxygen concentrations in the investigated groups.

\begin{tabular}{|c|c|c|c|}
\hline & $\mathrm{W}\left(\mathrm{O}_{2}\right), \%$ & $\mathrm{~W}\left(\mathrm{~N}_{2}\right), \%$ & $\begin{array}{l}\mathbf{W}\left(\mathbf{O}_{2}\right) / \\
\mathbf{W}\left(\mathbf{N}_{2}\right)\end{array}$ \\
\hline $\begin{array}{c}\text { Health } \\
\text { volunteers }\end{array}$ & $78.9 \pm 2.3$ & $21.1 \pm 1.3$ & $3.7 \pm 0.2$ \\
\hline $\begin{array}{l}\text { Cancer } \\
\text { patients }\end{array}$ & $75.2 \pm 1.5$ & $14.8 \pm 1.1$ & $5.1 \pm 0.4$ \\
\hline
\end{tabular}
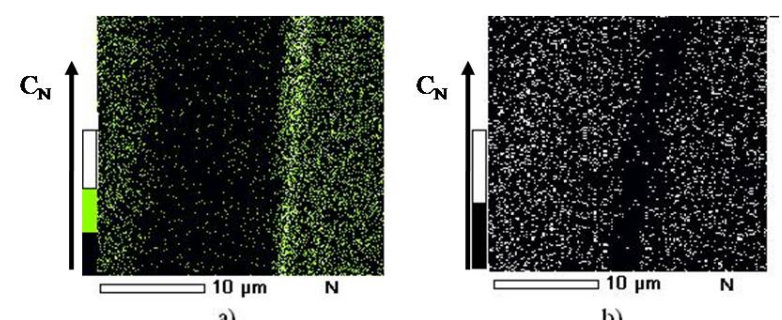

b)
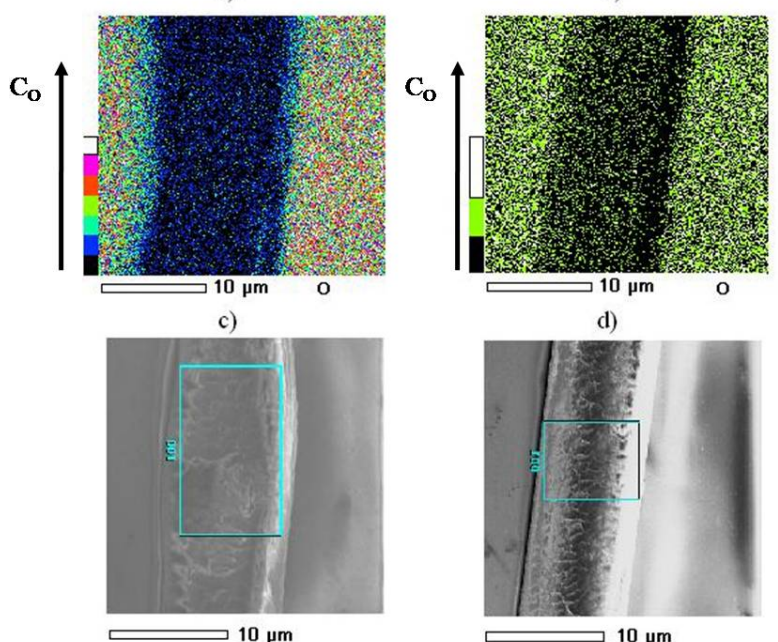

e)

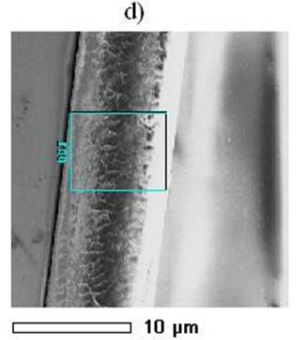

f)

Fig. 3 Map of nitrogen and oxygen distribution in external hair layer of healthy volunteers $(b, d, f)$ and cancer patients $(a, c, e)$. The y-axis shows the colour of the picture with increased element concentration.

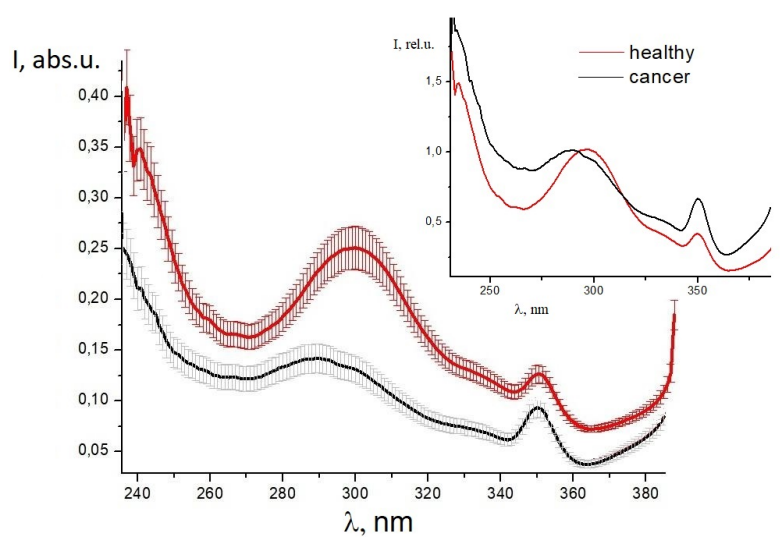

Fig. 4 The native (without processing) and normalized spectra of fluorescence excitation of extracted peptides from the external hair layer of healthy volunteers and cancer patients. The wavelength registration is $410 \mathrm{~nm}$.

In cells, the distribution of the microelements is heterogeneous, which can be seen in Fig. 3. The white dots correspond to the highest concentration of the registered microelement, while the black dots correspond to nil concentration. The dark stripe in the centre of Fig. 3 shows that the concentration there is minimum. In our opinion, this may be attributed to the features of energy distribution of electrons scattering from the hair cylindrical surface. Each microelement has a range of reflected electron energy. Since the 
reflected electron energy depends on the angle of scattering, the defined microelement concentration has its own spectrum and is heterogeneous through the entire hair surface. Thus, the microelement concentration should be compared with area with homogenous distribution (see the edge of the picture).

In healthy people, the distribution of oxygen and nitrogen is more homogenous (Fig. 3), but presence of malignant tumour leads to increased oxygen concentration, which may be due to increased concentration of reactive oxygen species. Change in nitrogen concentration for oncological patients has an adverse effect. It is known [9] that cancer cells assimilate hydrocarbons and peptides due to their own intensive metabolism. Nitrogen in cells transfers to other forms such as dioxide, ammonium ion, etc., which can be excreted from body. This leads to decreased nitrogen concentration. Moreover, as was proved in our previous work [10], data of external and internal layer can be experimentally observed due to the destruction of hair external layer in cancer patients.

The peptide impact on the results of SEM and their composition may be investigated by both laser flash photolysis and dynamic light scattering. However, we have found that hair has a strong intercellular matrix and, consequently, it is difficult to obtain cell suspension by hair fermentative treatment. Hence, peptides can be extracted from hair by treatment with carbonate buffer $(\mathrm{pH}=10)$. As a result of the procedure, the peptides are leached. This method, described in the work of Fedorkova et al. [11], finally leads to the solution of the dissolved peptides in buffer. In the result of measurements by the technique of dynamic light scattering, we did not receive a significant difference in the size of the dissolved peptides. Two types of peptides were registered with an average radius for hair in healthy volunteers of $3451 \pm 120 \mathrm{~nm}$ and $240 \pm 62 \mathrm{~nm}$. For cancer patients these means were $4132 \pm 154 \mathrm{~nm}$ and $234 \pm 58 \mathrm{~nm}$.

However, structure of peptides can be detected not only by dynamic light scattering but also by fluorimetry. For this purpose, the spectra of fluorescence excitation could be measured (Fig. 4). The wavelength registration was selected taking into account the region of absorbance and fluorescence of the most common peptides. As can be seen in the Fig. 4, these spectra are different and have several maxima such as at 295,
$350 \mathrm{~nm}$. For the cancer patients, the spectrum has a huge maximum at $295 \mathrm{~nm}$, but the spectrum for healthy volunteers has a small maximum at the wavelength and is shifting toward shorter wavelengths. We assume that the maximum at $350 \mathrm{~nm}$ is determined by the elastin contained in the hair [12]. The maximum at $295 \mathrm{~nm}$ is connected to the presence of tryptophan containing peptides [13], such as keratin [14]. It was found [8] that under the external layer of hair there is a Henley layer of keratin containing cells [15]. Since the disturbance of hair structure and the absence of the external layer are more expressed in the hair of cancer patients, the maximum at $295 \mathrm{~nm}$ is also more expressed for the hair of cancer patients.

\section{Conclusion}

Numerous investigations into hair structure and its element status touch provide data on peculiarities of hair for different nationalities [16] and age [5]. Our study revealed no significant difference in hair structure of people from different age groups. Moreover, the data about age-related changes in hair structure are controversial. In this respect, our data are close to the similar data obtained by another research team group [17] that showed insignificant age-related changes in hair structure for different age groups of the volunteers. Nonetheless, the possible age peculiarities are insignificant in comparison with the hair structure defects in cancer patients described in this work. The result of dynamic light scattering and fluorimetry demonstrate different peptides in hair structures of healthy volunteers and cancer patients. Registration of changes in peptides contribution could help to improve optical techniques of biomedical diagnostics of cancer.

\section{Disclosures}

All authors declare that there is no conflict of interests in this paper.

\section{Acknowledgments}

The authors greatly thank all the volunteers participating in the investigation and Ms. T. M. Tkacheva for their help in this work.

\section{References}

1. V. A. Kobliakov, "Mechanisms of tumor promotion by reactive oxygen species," Biochemistry 75(6), 675-685 (2010).

2. N. Shyh-Chang, "Metabolic Changes During Cancer Cachexia Pathogenesis," Advances in Experimental Medicine and Biology 1026, 233-249 (2017).

3. F. Briki, B. Busson, B. Salicru, F. Esteve, and J. Doucet, "Breast-cancer diagnosis using hair," Nature 400(6741), 226-227(1999). 
4. T. Pangršič, M. Gabrielaitis, S. Michanski, B. Schwaller, F. Wolf, N. Strenzke, and T. Moser, "EF-hand protein $\mathrm{Ca} 2+$ buffers regulate Ca2+ influx and exocytosis in sensory hair cells," Proceedings of the National Academy of Sciences 112(9), E1028-E1037 (2015).

5. M. Ambeskovic, E. Fuchs, P. Beaumier, M. Gerken, and G. A. Metz, "Hair trace elementary profiles in aging rodents and primates: links to altered cell homeodynamics and disease," Biogerontology 14(5), 557-567 (2013).

6. C. A. I. Ju, "Effect on Growth Performance and Hair Color in Weanling Piglets by Iron Glycine Chelate with Different Purity," Feed Review 3, 022 (2011).

7. S. B. Park, S. W. Choi, and A. Y. Nam, "Hair Tissue Mineral Analysis and Metabolic Syndrome," Biological Trace Element Research 130(3), 218-228 (2009).

8. Th. Schlake, "Determination of hair structure and shape," Seminars in Cell \& Developmental Biology 18(2), 267273 (2007).

9. N. M. Emanuel, R. E. Kavetskiy, B. N. Tarusov, and E. P. Sidorik, Biophysics of cancer, Science, Kiev (1976).

10. V. S. Maryakhina, M. V. Ovechkin, "Changes of oxygen status of hair cells for breast cancer presence," Lasers in medical science 31(5), 979-983(2016).

11. M. V. Fedorkova, N. V. Smolina, E. V. Mikhalchik, N. G. Balabushevich, G. A. Ibragimova, A. G. Gadzhigoroeva, E. I. Dmitrieva, and G. E. Dobretsov, "Effects of ultra violet radiation on the soluble proteins of human hair," Journal of Photochemistry and Photobiology B: Biology 140, 390-395 (2014).

12. J. E. Lai-Cheong, J. A. McGrath, "Structure and function of skin, hair and nails," Medicine, 41(6), 317-320 (2013).

13. J. R. Lakowicz, Principles of fluorescence spectroscopy, Springer Science \& Business Media (2013).

14. H. Kawasaki, M. Tominaga, A. Shigenaga, A. Kamo, Y. Kamata, K. Iizumi, U. Kimurac, H. Ogawa, K. Takamori, and F. Yamakura, "Importance of tryptophan nitration of carbonic anhydrase III for the morbidity of atopic dermatitis," Free Radical Biology and Medicine 73, 75-83 (2014).

15. W. Montagna, R. A. Ellis, The biology of hair growth, Academic Press, New York (2013).

16. N. Luther, M. E. Darvin, W. Sterry, J. Lademann, and A. Patzel, "Ethnic differences in skin physiology, hair follicle morphology and follicular penetration," Skin pharmacology and physiology 25(4), 182-191 (2012).

17. S. Nagase, Y. Kajiura, A. Mamada, H. Abe, S. Shibuichi, N. Satoh, T. Itou, Y. Shinohara, and Y. Amemiya, "Changes in structure and geometric properties of human hair by aging," International Journal of Cosmetic Science 60(6), 637-648 (2009). 